amine base is fairly uniform in regard to toxicity and sulfur content irrespective of the period of formation during the reduction.

Amino hydroxyarseno compounds in general contain the fewest sulfur atoms when prepared from the amino acids.

Fixation of the hydroxyl hydrogen in the nitrohydroxyarsonic acids tends to make the hydrosulfite reduction abnormal, and the products, when isolable, contain more sulfur than analogous substances prepared without fixation of this hydrogen atom. The hydroxyl hydrogen ortho to the nitro group seems to play an important role in the formation of arseno compounds of the type under consideration.

Probably the sulfonic acid group found in certain samples of arsphenamine enters the ring by way of the nitrogen atom with the intermediate formation of a sulfamic acid.

I wish to thank Dr. Reid Hunt for determining the toxicity of the samples involved in this work and Mr. Arthur J. Norton for his assistance in purifying the hydrosulfite and analyzing some of the substances obtained.

Boston, Massachusetts

[CONTRIBUtion fRom the FixED NITROgen Research LABORATORY]

\title{
MECHANISM OF GUANIDINE FORMATION IN FUSED MIXTURES OF DICYANODIAMIDE AND AMMONIUM SALTS
}

\author{
By J. S. Blatr and J. M. Brahaim ${ }^{1}$ \\ Received July 13, 1922
}

\section{Introduction}

In the course of an investigation on the use of calcium cyanamide as a source of nitroguanidine and other guanidine derivatives, some experiments were performed in which dicyanodiamide was fused with various ammonium salts, with the original purpose of determining the feasibility of this method for the production of guaridine salts. Their formation in this manner has been described by several investigators, but the optimum conditions for guanidine production had not at that time been ascertained. Our work along these lines was soon discontinued, ${ }^{2}$ however, since it was learned that the method was being investigated in another laboratory. ${ }^{3}$

However, our preliminary experiments had led us to a conception as to the reactions occurring on fusion of dicyanodiamide with ammonium

${ }^{1}$ Assistance in the analytical work given in this paper was rendered by C. D. Garby and L. A. Pinck.

2 The production of guanidine salts from calcium cyanamide by a more direct method was then studied and will form the subject of a later paper.

${ }^{3}$ Massachusetts Institute of Technology. See Davis, ThIs Journal, 43, 2234 (1921). 
salts which differs from that expressed in a recent article 4 by E. A. Werner and James $\mathrm{K}$. Bell. It was in an endeavor to throw some light on the chemistry of guanidine formation in fused mixtures that the further work described in this paper was performed.

\section{Reactions in the System, Dicyanodiamide-Ammonium Salt}

The product formed by fusing an ammonium salt with dicyanodiamide consists chiefly of guanidine and biguanide, both in the form of salts corresponding to the ammonium salt used, and a substance which though not definitely identified resembles ammeline (thio-ammeline in the case of the thiocyanate) or ammelide. ${ }^{5}$ The relative proportions of these constituents depend on the conditions of fusion.

To explain the formation of guanidine and biguanide under these conditions, Werner and Bell ${ }^{4}$ assume that dicyanodiamide first depolymerizes to form cyanamide which then reacts with the ammonium salt to give guanidine

$$
\left(\mathrm{H}_{2} \mathrm{CN}_{2}\right)_{2} \longrightarrow 2 \mathrm{H}_{2} \mathrm{NCN} ; \mathrm{H}_{2} \mathrm{NCN}+\mathrm{NH}_{3} \cdot \mathrm{HX} \longrightarrow \mathrm{H}_{2} \mathrm{NC}(\mathrm{NH}) \mathrm{NH}_{2} \cdot \mathrm{HX}
$$

and that biguanide is the product of a side-reaction in which 2 molecules of guanidine combine, with the loss of ammonia

$$
2 \mathrm{H}_{2} \mathrm{NC}(\mathrm{NH}) \mathrm{NH}_{2} . \mathrm{HX} \longrightarrow \mathrm{H}_{2} \mathrm{NC}(\mathrm{NH}) \mathrm{NHC}(\mathrm{NH}) \mathrm{NH}_{2} . \mathrm{HX}+\mathrm{NH}_{3} \mathrm{HX} \text {. }
$$

That dicyanodiamide does depolymerize under certain conditions of heating was first pointed out by Drechsel ${ }^{6}$ in 1875 , and recently by Werner and Bell. It has been further confirmed by experiments at this Laboratory. ${ }^{7}$ While depolymerization may take place to some extent on fusing dicyanodiamide with an ammonium salt, we have been entirely unsuccessful in attempts to demonstrate the presence of cyanamide during any stage of the fusion.

The assumption that biguanide results only from the combination of 2 molecules of guanidine, with liberation of 1 molecule of ammonia, appears to be contrary to the findings of other investigators and is entirely incompatible with the results of the present investigation. The basis for this assumption is evidently the fact that Bamberger and Dieckmann ${ }^{8}$ obtained biguanide hydrochloride in small yield by heating guanidine hydrochloride at $180-185^{\circ}$. These investigators, however, state in the same article that when guanidine thiocyanate ${ }^{9}$ was melted, the formation of biguanide was not demonstrable; it was, if formed, further decomposed.

+ Werner and Bell, J. Chem. Soc., 117, 1133 (1920).

${ }^{5}$ Klason, J. prakt. Chem., [II] 33, 290 (1886).

- Drechsel, Dissertation, Univ. of Leipzig, Apr. 25, 1875.

${ }^{7}$ We have found that when the vapors, formed by heating dicyanodiamide above its melting point until a violent reaction sets in, are condensed on a cold surface, the condensate contains some cyanamide.

${ }^{8}$ Bamberger and Dieckmann, Ber, 25, 543 (1892).

${ }^{9}$ Ammonium thiocyanate and dicyanodiamide were used by Werner and Bell. 
The mechanism of guanidine formation in fused mixtures of dicyanodiamide and an ammonium salt which was suggested by the results of our preliminary experiments and which appears to be fully confirmed by the results described in the present paper, consists first in the formation of the biguanide salt by the addition of the ammonium salt to the nitrile group of dicyanodiamide.

$\mathrm{H}_{2} \mathrm{NC}(: \mathrm{NH}) \mathrm{NHCN}+\mathrm{NH}_{3} \cdot \mathrm{HX} \longrightarrow \mathrm{H}_{2} \mathrm{NC}(: \mathrm{NH}) \mathrm{NHC}(\mathrm{NH}) \mathrm{NH}_{2} . \mathrm{HX}$.

A second molecule of ammonium salt then adds to form 2 molecules of the guanidine salt.

$$
\mathrm{H}_{2} \mathrm{NC}(\mathrm{NH}) \mathrm{NHC}(\mathrm{NH}) \mathrm{NH}_{2} . \mathrm{HX}+\mathrm{NH}_{3} \cdot \mathrm{HX} \longrightarrow 2 \mathrm{H}_{2} \mathrm{NC}(\mathrm{NH}) \mathrm{NH}_{2} . \mathrm{HX} \text {. }
$$

This mechanism has not, to our knowledge, been previously suggested. ${ }^{10}$

The first reaction, biguanide formation, has long been known and has been described by Bamberger and Dieckmann, ${ }^{8}$ Smolka and Friedrich, ${ }^{11}$ and others. The second reaction has not hitherto been described. ${ }^{10}$ It is interesting to note in this connection that the reactions taking place in fused mixtures of dicyanodiamide and ammonium salts are in accord with E. C. Franklin' ${ }^{12}$ ammono-carbonic acid classification of these compounds, and that another step in the melon-to-guanidine ammonation series has now been accomplished. ${ }^{13}$

The experimental evidence for the validity of our assumption as to the mechanism of guanidine formation is based principally on 3 experiments. In one, dicyanodiamide was fused with ammonium thiocyanate under the same conditions as those maintained by Werner and Bell, but samples of the melt were taken at intervals during the fusion as well as of the original mixture and final melt. These samples were analyzed for total nitrogen, ammonia, guanidine and biguanide, and qualitatively tested for cyanamide. A similar experiment was performed using ammonium nitrate instead of ammonium thiocyanate. In the third experiment, biguanide nitrate was fused with ammonium nitrate. Qualitative evidence as to the course of the reaction was also obtained in experiments in which dicyanodiamide was fused with ammonium sulfate and with ammonium chloride.

The results of the quantitative experiments are given in the tables to follow, and the course of the reaction in the case of ammonium thiocyanate is shown in Fig. 1. In connection with these results attention is called to the following points.

That biguanide thiocyanate is formed by the addition of ammonium thiocyanate to dicyanodiamide, rather than from 2 molecules of guanidine thiocyanate, with loss of a molecule of the ammonium salt, is shown by

${ }^{10}$ See, however, Davis, Ref. 3, p. 2235.

${ }^{11}$ Smolka and Friedrich, Monatsh., 10, 86 (1889); 9, 228 (1888).

${ }_{12}$ Franklin, This Journal, 44, 486 (1922).

13 Ref. 12, p. 489. 
the fact that this salt was formed in the system in considerable percentages long before the guanidine salt was formed. In all of the experiments its concentrations rose quite rapidly to a maximum value and then more gradually decreased, being present in the final melts only in very small concentrations.

That guanidine nitrate is formed by the addition of ammonium nitrate to biguanide nitrate is conclusively shown by the experiment in which the 2 latter salts were fused together. There is every reason to believe that a similar reaction occurs with the other ammonium salts. In Fig. 1, for

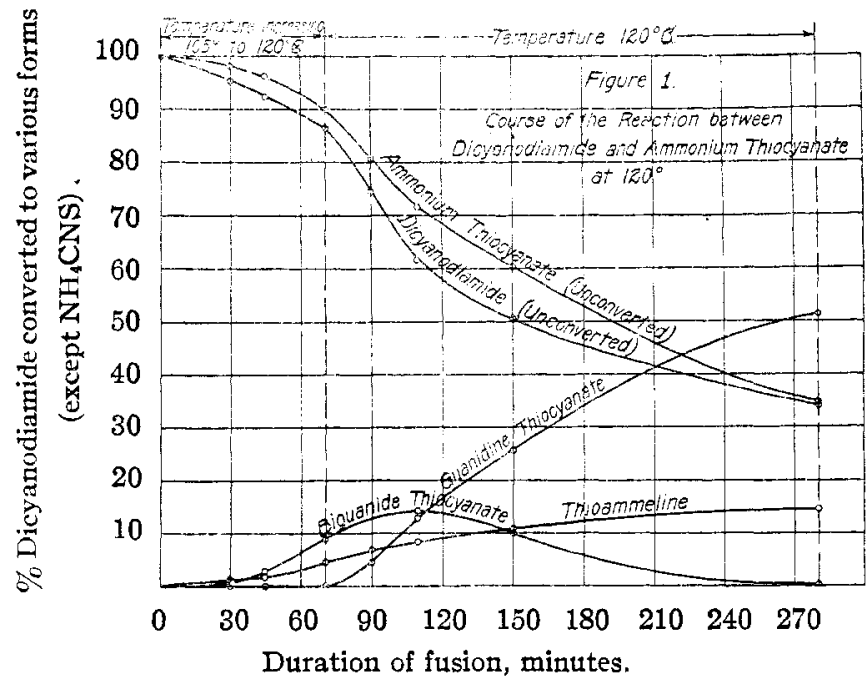

Fig. 1.-Course of the reaction between dicyanodiamide and ammonium thiocyanate at $120^{\circ}$.

example, it is seen that guanidine did not make its appearance in the system until the concentration of biguanide had nearly reached its maximum value, and that the concentration of guanidine steadily increased while the concentration of biguanide simultaneously decreased. It will also be noted in Fig. 1 that the concentration of ammonium thiocyanate fell off rapidly after guanidine began to form, combining not only with the dicyanodiamide but also with the biguanide. As will be explained later, the analytical data for dicyanodiamide are not as reliable as for the other constituents and hence an interpretation of the course of the reaction based on them is not fully justified. They are, however, in general agreement with the other data.

While it is possible that a part of the guanidine may be formed by the addition of the ammonium salt to depolymerized dicyanodiamide, we have, as previously mentioned, been unable to obtain any proof for it. On the other hand, the experimental results can be satisfactorily explained on the assumption of the formation of biguanide as an intermediate compound. 
As to the loss of ammonia from the system and as to the formation of thio-ammeline, it appears that, as suggested by Werner and Bell, ${ }^{4}$ a part of the ammonium thiocyanate is decomposed into ammonia and thiocyanic acid, the latter compound then reacting with dicyanodiamide to form thio-ammeline and the ammonia being evolved. Our values for total nitrogen and thio-ammeline nitrogen substantiate this view for while the ammonia loss during the first part of the experiment was less than that corresponding to the thio-ammeline formed, this apparent discrepancy may be explained on the basis of the retention of free ammonia by the somewhat viscous melt, which later becomes quite fluid. In this connection it may be mentioned that the statement ${ }^{14}$ that ammonia is liberated from a fused melt of dicyanodiamide and ammonium nitrate due to the reaction of ammonium nitrate with biguanide nitrate to form a biguanide salt with 2 equivalents of acid is probably in error, and is perhaps traceable to Rathke. ${ }^{15}$

Werner and $\mathrm{Bell}^{4}$ are led to the rejection of the cyanoguanidine formula, $\mathrm{H}_{2} \mathrm{NC}(: \mathrm{NH}) \mathrm{NHCN}$, for dicyanodiamide on the basis of their interpretation of the reactions occurring in fused mixtures of dicyanodiamide and ammonium salts, and state that the formation of thio-ammeline on fusion of dicyanodiamide with ammonium thiocyanate is "difficult to explain on the basis of the cyanoguanidine structure for dicyanodiamide." It appears, however, that our results are in complete accord with and evidence for the cyanoguanidine formula. The addition of ammonia to dicyanodiamide to form biguanide is a reaction involving the nitrile group of cyanoguanidine. The addition of thiocyanic acid to dicyanodiamide, followed by ring closure to form thio-ammeline, may also be interpreted as being in entire accord with the cyanoguanidine formula. The mechanism may be as follows.

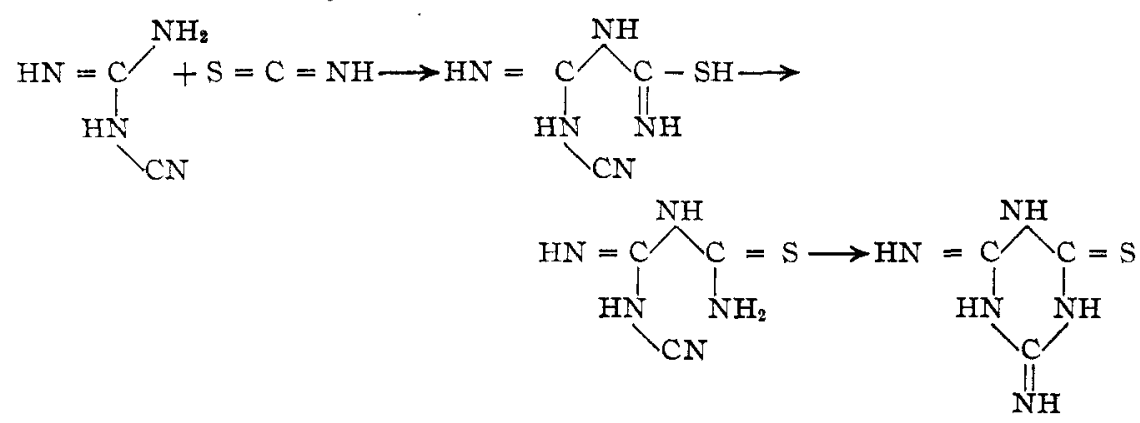

${ }^{14}$ Ref. 3, p. 2235.

15 Rathke, Ber., 12, 776 (1879). We have found that a biguanide salt containing 1 equivalent of acid gives a practically neutral aqueous solution. Salts with 2 equivalents of a strong acid, such as $\mathrm{C}_{2} \mathrm{H}_{1} \mathrm{~N}_{5}\left(\mathrm{NO}_{3}\right)_{2}$, are strongly acid in reaction. See also Rachmann, Ann., 376, 113 (1910). 
Although we have no direct experimental basis for this suggestion, reactions analogous to each of the assumed steps are well known. It is seen that the reactions studied in the present investigation simply add somewhat to the already overwhelming evidence ${ }^{16}$ as to the correctness of the cyanoguanidine structure for dicyanodiamide.

\section{Analytical Methods}

Guanidine.-Guanidine was estimated by the picrate method described by $A$. Vozarik. ${ }^{17}$ We have found that the method is capable of giving excellent results when the ammonium picrate concentration of the solution from which the guanidine picrate separates is properly regulated. By saturating the Gooch filter with the wash-solution before the initial drying and weighing, the necessity for making Vozarik's "asbestos correction" can be avoided. The method is open to the objection, however, that biguanide and guanylurea, compounds often occurring with guanidine, give fairly insoluble picrates under the same conditions as does guanidine. Guanylurea was not present in any of the samples analyzed in this investigation.

Biguanide picrate is much more soluble in ammonium picrate solutions than is guanidine picrate, and is also easily distinguished from the latter compound by its appearance, and by the slowness with which it is formed. Guanidine, even when present in low concentration, is practically immediately precipitated in fine, light yellow crystals upon the addition of the Vozarik reagent. On the other hand, even in fairly concentrated solutions of biguanide, the picrate of the latter substance is only very slowly precipitated-not at all quantitatively-as long, thin rods, while in dilute solutions biguanide picrate does not precipitate at all. When present in small amounts, therefore, it affects the accuracy of guanidine determinations only very slightly. This was the case in most of our work, and when otherwise, corrections were applied which are described under the individual experiments.

Biguanide.-Biguanide was estimated by a method which will form the subject of a later report from this laboratory. It is based on the insolubility of nickel biguanide in alkaline solution. Its accuracy is shown by an experiment in which 3 samples of pure biguanide nitrate (m. p. 201.5-201.7 corr.) of 0.1 g. each gave precipitates weighing $0.0790,0.0787$ and 0.0788 , corresponding to $100.25,99.86$ and $100.00 \%$ recovery.

Dicyanodiamide.-The method developed at this laboratory for the determination of dicyanodiamide, ${ }^{18}$ based on its conversion to guanylurea and precipitation as a nickel salt, was not found to be applicable in the thiocyanate experiment. In the presence of thiocyanates, dicyanodiamide is converted by the action of dil. acids not only to guanylurea but also to thio-ammeline. Attempts to remove the thiocyanate first were unsuccessful. In this one experiment, therefore, the dicyanodiamide was estimated by difference between total nitrogen and nitrogen in forms other than dicyanodiamide. The dicyanodiamide values are consequently subject to greater error than those of the other constituents.

Ammonia.-Ammonia was determined by an aeration method described by Mathews ${ }^{19}$ since it was found that the distillation of samples containing such compounds as guanidine and biguanide with even such a weak base as magnesium oxide resulted in a partial hydrolysis with evolution of ammonia. In a trial experiment using ammo-

16 Bamberger, Ber., 16, 1460 (1883); 23, 1857 (1890). Ref. 11. Poh1, J. prakt. Chem., 77, 553 (1908). Especially Jakuschkin, Ann. Inst. Agr. Moscow, 15, 176 (1909).

17 Vozarik, $Z$. angew. Chem., 15, 670 (1902).

${ }_{18}$ To be published soon.

${ }^{19}$ Mathews, J. Agr. Sci., 10, 72 (1920). 
nium sulfate, the ammonia obtained from 2 samples by aeration was 99.71 and $100 \%$ of the ammonia obtained by distillation.

Thio-ammeline.-Thio-ammeline is insoluble in water but soluble in alkaline solutions. The thio-ammeline in the melts was, therefore, determined by treating the entire sample with water, neutralizing the slightly alkaline solution to reduce the solubility of thio-ammeline, filtering through a tared Gooch crucible, washing the insoluble material well with water and drying and weighing the crucible. The weighed material was then treated with $10 \%$ potassium hydroxide solution which dissolved the thioammeline, and the weight of the alkali-insoluble material was determined. The waterinsoluble, alkali-soluble material was considered as thio-ammeline, allowance being made for the small amount of material of similar solubility found in the unfused mixture. One sample of thio-ammeline thus isolated contained $49.39 \%$ of nitrogen, while that calculated in thio-ammeline is $49.00 \%$.

\section{Description of Experiments}

Fusion of Dicyanodiamide with Ammonium Thiocyanate.-An experiment was performed in which an effort was made to closely duplicate the experiment by Werner and Bell, previously referred to. Double the quantity of dicyanodiamide and ammonium thiocyanate was used, however, so as not to change the conditions of the experiment materially by the withdrawal of samples during the fusion.

In this experiment, then, an intimate mixture consisting of $84 \mathrm{~g}$. of dicyanodiamide $(99.8 \%$ pure) and $152 \mathrm{~g}$. of ammonium thiocyanate which had been dried for several hours at $105^{\circ}$ was placed in a beaker and heated on an oil-bath. It began to melt at $105^{\circ}$ instead of at $80^{\circ}$ as found by Werner and Bell. Complete liquefaction took place on maintaining the mixture at this temperature for about 30 minutes, and the temperature was then raised to $120^{\circ}$ during a period of 40 minutes, thus reaching this temperature 70 minutes after the mixture began to melt. The melt was then maintained at this temperature for 3.5 hours.

The samples, 8 in all, were treated with water, the water-insoluble material (thioammeline) was filtered off and the various constituents in the filtrate determined by the methods just described. The results of these analyses are expressed in the form of percentage conversion and plotted as a function of the duration of fusion in Fig. 1. The guanidine values at the end of 90 and 110 minutes may be somewhat too high, due to the presence in these samples of biguanide in relatively high concentrations as compared with guanidine. This possible error could not materially affect the interpretation of the course of the reaction. Our interpretation of these results has already been stated.

It is seen that the reaction was incomplete at this temperature, the yield of guanidine being only about $52 \%$. It is probable that a better yield of guanidine could be obtained by employing a higher temperature but since the method is only of minor importance as a method of guanidine preparation, further work was not done along this line. In this connection it may be mentioned that Werner and Bell ${ }^{20}$ reported a $90.8 \%$ yield of guanidine thiocyanate in an experiment under the same conditions as above described. The discrepancy may be explained by the fact that

${ }^{20}$ Ref. 4, p. 1135. 
their analytical method, ${ }^{21}$ as described, does not differentiate between guanidine thiocyanate on the one hand and a mixture of dicyanodiamide and ammonium thiocyanate (in molecular proportions of 1 to 2 ) on the other hand. The percentage of thiocyanate in the two cases is the same and no evidence is offered to show that the "guanidine thiocyanate" isolated by them was a pure compound.

Fusion of Dicyanodiamide with Ammonium Nitrate.-An intimate mixture consisting of $100 \mathrm{~g}$. of dicyanodiamide and $190 \mathrm{~g}$. of ammonium nitrate (molecular proportions, 1 to 2 ) was heated under the same conditions as in the preceding experiment except that a higher temperature was used. In a preliminary experiment in which the mixture was heated at $135^{\circ}$ for 90 minutes it was found that biguanide was formed to a considerable extent but only a very small quantity of guanidine was formed.

A similar mixture was then heated up to $150^{\circ}$ during a period of 55 minutes and held at that temperature for 90 minutes, at the end of which the melt had completely solidified to a hard, dense mass. Six samples were taken during the heating and analyzed only for biguanide and ammonia. The results are shown in the following table.

TABLE I

\begin{tabular}{|c|c|c|c|c|}
\hline RESUL & USION OF DIC & ANODIAMI & WITH AMMONIU & Nitrate \\
\hline Sample & $\begin{array}{l}\text { Duration of } \\
\text { heating }\end{array}$ & $\begin{array}{l}\text { Temp. of } \\
\text { system }\end{array}$ & $\begin{array}{l}\mathrm{NH}_{4} \mathrm{NO}_{3} \text { (calc. } \\
\text { from } \mathrm{NH}_{3} \text { ) }\end{array}$ & $\begin{array}{c}\text { Biguanide } \\
\text { nitrate }\end{array}$ \\
\hline 1 & Min. & ${ }^{\circ} \mathrm{C}$. & $\%$ & $\%$ \\
\hline $\begin{array}{l}1 \\
2\end{array}$ & 50 & 146 & 59.3 & 9.85 \\
\hline 3 & 75 & 150 & 36.9 & 15.4 \\
\hline 4 & 90 & 150 & 29.6 & 13.4 \\
\hline 5 & 110 & 150 & 24.5 & 12.4 \\
\hline 6 & 145 & 150 & $\ldots$ & 6.84 \\
\hline
\end{tabular}

Through an error Sample 6 was not analyzed for $\mathrm{NH}_{3}$.

a The ammonia analyses are all somewhat too high, except for Sample 1, due to the fact that these analyses were made by distillation of the solutions made alkaline with magnesium oxide. It was subsequently found that biguanide decomposes under such treatment with evolution of ammonia.

It is seen that the results corroborate the conclusions drawn from the thiocyanate experiment as to biguanide formation. The reaction clearly was no more complete in this case than in the experiment in which ammonium thiocyanate was used, which is perhaps to be attributed in this case, as in the other, to the fact that the temperature was not high enough. One point of difference between the two, however, is that in the case of

${ }^{21}$ The reaction product was dissolved in water, filtered from water-insoluble substance, mainly thio-ammeline, and the filtrate evaporated to dryness. The residue was again taken up with water and the solution filtered free from more thio-ammeline. The filtrate was evaporated at $100^{\circ}$ "to a sirupy consistency" and when cooled, this "set to a crystalline mass." This material was analyzed for thiocyanate and the result was in close agreement with the thiocyanate required for pure guanidine thiocyanate. 
the nitrate, the melt solidified toward the end of the experiment whereas in the case of the thiocyanate the melt, although at a much lower temperature, remained fluid throughout. This difference, due of course to the difference in melting point of guanidine nitrate and guanidine thiocyanate, probably explains the fact that in the case of the nitrate the biguanide did not completely disappear, premature solidification of the melt tending to inhibit the reaction.

Fusion of Biguanide Nitrate with Ammonium Nitrate.-To obtain additional evidence as to the second step in the formation of guanidine from dicyanodiamide, namely, the transformation of biguanide to guanidine, an experiment was performed in which biguanide nitrate was fused with ammonium nitrate. The biguanide used was $98.5 \%$ pure by our method of analysis and contained no guanidine.

Twenty $\mathrm{g}$. of this material and $9.76 \mathrm{~g}$. of ammonium nitrate (equimolecular proportions) were well mixed and fused in a manner similar to that in the previous experiment. The mixture was heated to $150^{\circ}$ during a period of 25 minutes, and maintained at that temperature for 85 minutes. Toward the end of the heating, the system became more and more pasty and finally quite solid, behaving in this respect similarly to the ammonium nitrate-dicyanodiamide mixture. The latter mixture began to fuse at $120^{\circ}, 20^{\circ}$ lower than the fusion temperature of the ammonium nitrate-biguanide nitrate mixture.

The material was analyzed before and after fusion. Before fusion (A) $34.3 \%$ of ammonium nitrate, $66.2 \%$ of biguanide nitrate and no guanidine nitrate, were found. while after fusion (B) $12.4 \%, 29.5 \%$ and $54.2 \%$, respectively, of these substances were recovered.

The result given here for guanidine requires explanation. Although picrates were obtained from both $A$ and $B$, the absence of guanidine before fusion and presence after fusion was easily shown by the appearance of the picrates. A sample before fusion weighing $0.2000 \mathrm{~g}$. gave $0.1956 \mathrm{~g}$. of biguanide picrate. The theoretical yield is 0.2665 g., showing that precipitation is not quantitative. However, using this result and the biguanide analyses before and after fusion a correction to be subtracted from the total picrate obtained after fusion was calculated, which gave $0.2555 \mathrm{~g}$. as the net weight of guanidine picrate from $0.2000 \mathrm{~g}$. after fusion. This corresponds to $0.1081 \mathrm{~g}$. of guanidine nitrate, or $54.2 \%$. The ammonia analyses here were by aeration, and are quite dependable.

The presence of guanidine in the product was further confirmed by the isolation of a material, melting at $203-210^{\circ}$ (uncorr.) (m. p. of pure guanidine nitrate is $215.5-216.5^{\circ}$ corr.) , which had the very characteristic appearance, under the microscope, of guanidine nitrate. The impurity consisted of the material referred to below as ammeline or ammelide.

There was also isolated from the material after fusion an appreciable quantity of a white material, soluble in alkaline and in strongly acid solutions, and in other respects resembling ammeline or ammelide. ${ }^{5}$ Sim- 
ilar material was also always obtained in the products of fusion of dicyanodiamide with ammonium nitrate, chloride, and sulfate. Because of the difficulty of separation from guanidine salts, it is always present as an impurity in crude guanidine salts prepared in this manner. We have not studied this material except that a nitrogen determination was made on the product isolated from the fusion of dicyanodiamide and ammonium sulfate, described below. The determination showed $51.27 \%$ nitrogen, while the theoretical value for ammeline is $55.11 \%$ and for ammelide, $43.77 \%$. It does not form an insoluble picrate and therefore does not interfere with the guanidine determination.

In this connection it may be mentioned that Bamberger and Dieckmann ${ }^{8}$ reported the formation of ammeline, $\mathrm{C}_{3} \mathrm{H}_{5} \mathrm{~N}_{5} \mathrm{O}$, on fusion of guanidine hydrochloride. The basis of their identification was its solubility, referred to above. It will be noted that the latter compound contains no oxygen so that its transformation to an oxygen-containing compound merely by fusion is difficult to explain. A study is now being made at this Laboratory of the so-called ammeline-ammelide material obtained under various conditions with a view to ascertaining its composition and properties.

Fusion of Dicyanodiamide with Ammonium Chloride and with Ammonium Sulfate.-Experiments on the fusion of dicyanodiamide with ammonium chloride and with ammonium sulfate were performed to gain an idea of the character of the reactions taking place and of the conditions of fusion. Small quantities were used, and only qualitative tests were made during and after the fusion. The principal significance of these experiments consists in showing that in the case of these salts also, the concentration of biguanide rose rapidly to a maximum during the first part of the fusion, and then more slowly decreased. It was found, however, that with these 2 salts a higher temperature was required for the fusion to take place. As a result the exothermic character of the reaction became more apparent, and a greater quantity of by-products was formed, especially the material called, by Bamberger, ammeline.

The work with these two salts was done before the appearance of the recent article by Ewan and Young. ${ }^{22}$ It is interesting to note that they found it desirable from the point of view of guanidine preparation to raise the temperature toward the end of the reaction, which is in accord with our observation that prolonged heating at moderate temperatures results in only partial conversion to guanidine.

\section{Summary}

1. The following mechanism of the formation of guanidine salts in ${ }^{22}$ Ewan and Young, J. Soc. Chem. Ind., 40, 109 (T), (1921). 
fused mixtures of dicyanodiamide and ammonium salts, is suggested and experimentally verified.

I. $\mathrm{H}_{2} \mathrm{NC}(: \mathrm{NH}) \mathrm{NHCN}+\mathrm{NH}_{3} \cdot \mathrm{HX} \longrightarrow \mathrm{H}_{2} \mathrm{NC}(: \mathrm{NH}) \mathrm{NHC}(: \mathrm{NH}) \mathrm{NH}_{2} \cdot \mathrm{HX}$

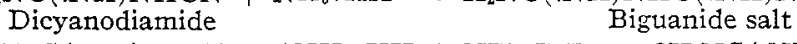

II. $\mathrm{H}_{2} \mathrm{NC}(: \mathrm{NH}) \mathrm{NHC}(: \mathrm{NH}) \mathrm{NH}_{2} \cdot \mathrm{HX}+\mathrm{NH}_{3} \cdot \mathrm{HX} \longrightarrow 2 \mathrm{H}_{2} \mathrm{NC}(: \mathrm{NH}) \mathrm{NH}_{2} \cdot \mathrm{HX}$ Biguanide salt

Guanidine salt

2. The mechanism previously suggested, postulating the depolymerization of dicyanodiamide in such fused mixtures is not capable of direct experimental verification.

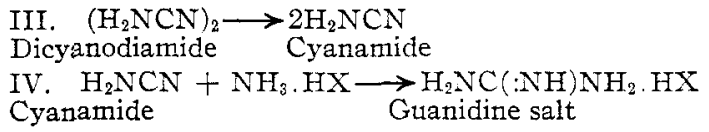

3. The statement that biguanide salts are formed in such mixtures by de-ammonation of 2 molecules of guanidine salt (reverse of Reaction II, above) is shown to be very doubtful. The salt of biguanide is intermediate in the formation of guanidine salts in such fused mixtures, and is not a product of a side reaction.

4. It is shown that the reactions taking place in such fused mixtures cannot be offered as evidence against the cyanoguanidine structure for dicyanodiamide, and are rather in entire accord with it.

Washington, D. C.

[CONTRIBUTION FROM THE ChEMical Laboratories OF Columbia University]

RESEARCHES ON SELENIUM ORGANIC COMPOUNDS. I. SYNTHESIS OF 2-METHYL-4-SELENOQUINAZOLONE, 2-PHENYL-BENZOSELENAZOLE, AND SOME DERIVATIVES OF THE LATTER ${ }^{1}$

\author{
By Marston Taylor Bogert and YÜ-Gwan Chen ${ }^{2}$ \\ Received July 15, 1922
}

\title{
Introductory
}

Since metallic selenium is now available in considerable amount and at a reasonable price, it seemed to the authors an appropriate time to investigate somewhat further the possibilities of discovering organic selenium derivatives of service to mankind as drugs or dyes, or perhaps in still other fields.

The powerful physiological action of hydrogen selenide is well known. Valuable therapeutic properties have been claimed from time to time for various organic selenium compounds but, so far as we are aware, none of these substances are used in medicine to-day.

1 The $o$-nitro-aniline used in this investigation was provided by the National Anjline and Chemical Co., and the selenium by the Baltimore Copper Smelting and Rolling Co., and we welcome this opportunity of expressing to them publicly our appreciation of their courtesy.

${ }^{2}$ Read at the Birmingham meeting of the American Chemical Society, April, 1922. 